\title{
AIGaAs inverted strip buried heterostructure lasers
}

\author{
H. Blauvelt, S. Margalit, and A. Yariv \\ California Institute of Technology, Pasadena, California 91125
}

(Received 7 May 1982; accepted for publication 14 June 1982)

\begin{abstract}
Inverted strip buried heterostructure lasers have been fabricated. These lasers have threshold currents and quantum efficiencies that are comparable to those of conventional buried heterostructure lasers. The optical mode is confined by a weakly guiding strip loaded waveguide which makes possible operation in the fundamental transverse mode for larger stripe widths than is possible for conventional buried heterostructure lasers. Scattering of the laser light by irregularities in the sidewalls of the waveguide, which can be a serious problem in conventional buried heterostructure lasers, is also greatly reduced in these lasers.
\end{abstract}

PACS numbers: 42.55.Px, 42.80.Sa

Large optical cavity buried heterostructure (LOCBH) lasers have recently been developed by Chinone et al. ${ }^{1}$ which have many highly desirable characteristics. These lasers have low threshold currents (8-10-mA/ $\mu \mathrm{m}$ stripe width), high differential quantum efficiencies $(60-80 \%)$, and they operate stably in a single transverse and longitudinal mode. However, fundamental transverse mode operation is achievable only for relatively narrow stripe widths. The maximum allowable stripe width is typically between 2 and $3 \mu$ depending on the exact composition of the layers. As a result the power output of these lasers is limited by catastrophic mirror damage to approximately $20 \mathrm{~mW} \mathrm{cw}$ and $80 \mathrm{~mW}$ for 100 -ns pulses. Scattering of light by irregularities in the sidewalls of the waveguide can also result in a significant degradation of beam quality. This is of particular importance for lasers with stripe widths less than $2 \mu .^{2} \mathrm{~A}$ second important type of buried heterostructure laser is the strip buried heterostructure (SBH) laser developed by Tsang and Logan. ${ }^{3}$ These lasers can be operated in a stable fundamental mode for strip widths up to $5 \mu$ and are much less susceptible to degradation of beam quality due to scattered light. However, fabrication of this laser requires epitaxial growth on top of an $\mathrm{Al}_{x} \mathrm{Ga}_{1-x}$ As guide layer. To obtain good growth on top of the guide layer requires a precisely controlled meltback prior to the second liquid phase epitaxial (LPE) growth. By comparison the layers of the second LPE growth of the LOCBH lasers are grown starting from the GaAs substrate and can be simply and reliably grown without a meltback. In this letter we report the fabrication of inverted strip buried heterostructure lasers (ISBH), which combine many of the desirable features of the LOCBH and SBH lasers.

The ISBH laser structure is shown schematically in Fig. 1. The structure resembles that of the LOCBH laser except the active and the lower cladding layers are narrower in width than the $\mathrm{Al}_{0.24} \mathrm{Ga}_{0.76}$ As optical guide layer. This results in two significant changes in the modal characteristics of the laser. First, the laser mode is isolated from the sidewalls of the optical guide layer, in which most of the power propagates. This greatly reduces irregularities in the far-field patterns of the lasers due to scattered light. Second, the confinement of the mode in the lateral direction by a weakly guiding strip loaded waveguide makes possible operation of these lasers in the fundamental transverse mode for wider stripe widths than can be achieved for LOCBH lasers.
The fabrication of ISBH lasers requires two LPE growths. The layers grown in the first growth are an $N$-Al $\mathrm{l}_{0.5} \mathrm{Ga}_{0.5}$ As bottom cladding layer $(1.5 \mu \mathrm{m}$ thick, $\mathrm{Te}$ doped), an $\mathrm{Al}_{0.05} \mathrm{Ga}_{0.95} \mathrm{As}$ active layer $(0.10 \mu \mathrm{m}$ thick, undoped), a $P-\mathrm{Al}_{0.24} \mathrm{Ga}_{0.76}$ As optical guide layer $(0.8 \mu \mathrm{m}$ thick, Ge doped), and a $P$ - $\mathrm{Al}_{0.37} \mathrm{Ga}_{0.63}$ As top cladding layer (1.0 $\mu \mathrm{m}$ thick, Ge doped). After the first growth, mesas are formed by etching down to the GaAs substrate using $\mathrm{H}_{2} \mathrm{SO}_{4}: \mathrm{H}_{2} \mathrm{O}_{2}: \mathrm{H}_{2} \mathrm{O}$ 1:8:8. The width of the guide layer after the mesa etch, $W_{1}$, is typically $5-7 \mu$. Next the bottom cladding layer is selectively etched in an HF etch until the top of the mesa is undercut by $1-1.5 \mu$. The portion of the active layer which is exposed after the HF etch is then selectively removed using $\mathrm{H}_{2} \mathrm{O}_{2}(p \mathrm{H}=7.0)$. The width of the remaining portion of the active layer, $W_{2}$, is typically $2.5-4 \mu$. Next a thin $P-\mathrm{Al}_{0.6} \mathrm{Ga}_{0.4}$ As layer and an $N-\mathrm{Al}_{y} \mathrm{Ga}_{1-y}$ As layer are grown in the second LPE growth. As in the case of the LOCBH lasers, these layers are grown starting from the $\mathrm{GaAs}$ substrate. A meltback step is not required, nor are there any limitations on the aluminum content of the optical guide layer. Figure 2 is a scanning electron microscope photograph of a cleaved cross section of an ISBH laser.

Normally, LOCBH lasers are grown with $N$-type optical guide layers to minimize electron leakage. When $n$-type GaAs substrates are used this means the active layer is on top of the guide layer. However, it is difficult to fabricate these lasers with the active layer on top of the guide layer, since this would require growing over the $\mathrm{AlGaAs}$ guide layer in the second LPE growth. Having the active layer underneath the optical guide layer enables the layers of the second growth to be grown starting from the GaAs substrate. ISBH

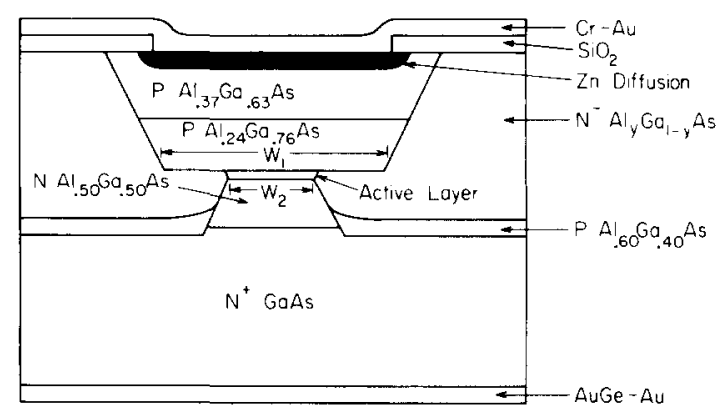

FIG. 1. Schematic diagram of a cross section of an ISBH laser. 

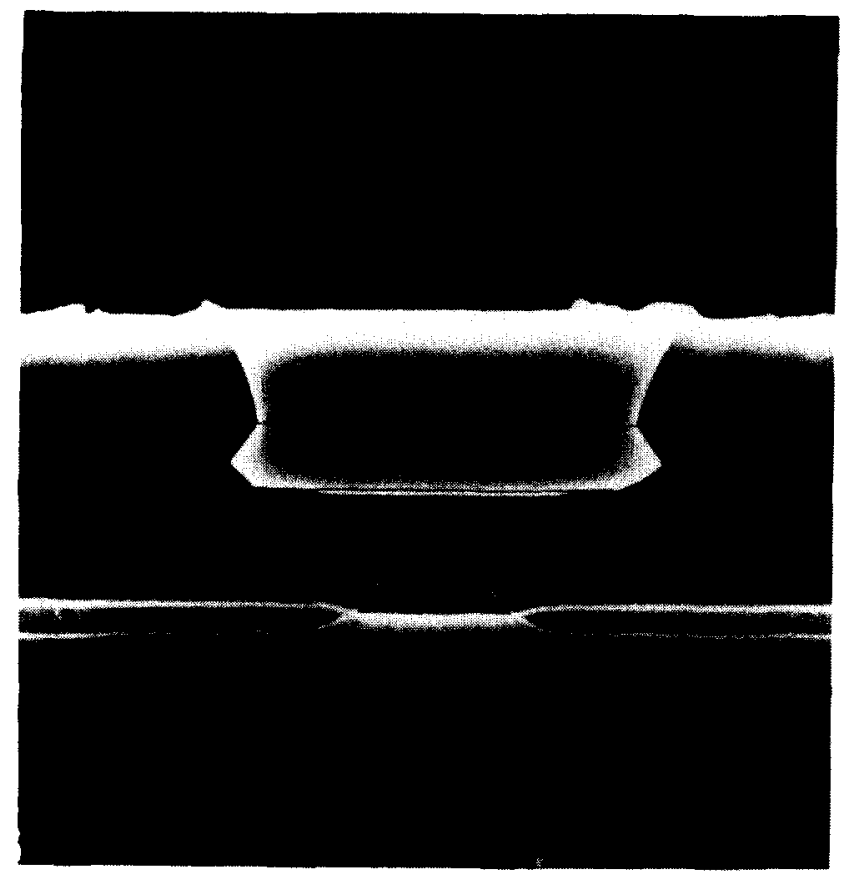

FIG. 2. Scanning electron micrograph of a cleaved cross section of an ISBH laser.

lasers can still have $N$-type optical guide layers if $p$-type GaAs substrates are used.

ISBH lasers were found to have threshold currents and differential quantum efficiencies that were nearly identical to those of LOCBH lasers fabricated in our laboratory. For $300-\mu \mathrm{m}$ cavity lengths, pulsed threshold currents were typically $25-30 \mathrm{~mA}$ for $2.5-\mu \mathrm{m}$-wide active layers and $35-40 \mathrm{~mA}$ for $5-\mu \mathrm{m}$-wide active layers. $\mathrm{cw}$ thresholds were approximately $10 \%$ higher. Differential quantum efficiencies were generally $50-60 \%$ and exceeded $70 \%$ in a few devices. ISBH lasers operated stably in a single transverse and longitudinal mode. For lasers with $\mathrm{Al}_{0.3} \mathrm{Ga}_{0.7}$ As burying layers ISBH lasers operated predominantly in the fundamental transverse mode for active layer widths $W_{2}$, up to $4 \mu$. By comparison, LOCBH lasers fabricated in our laboratory operated predominantly in the fundamental mode only for active layer widths less than $2 \mu$. Since fundamental mode operation of LOCBH lasers for stripe widths greater than $2 \mu$ has been achieved elsewhere, ${ }^{2}$ it is possible that predominantly fundamental mode operation of ISBH lasers with stripe widths greater than $4 \mu$ can also be achieved by optimizing this structure. A second important characteristic of ISBH lasers was that irregularities in the far-field patterns of the lasers were greatly reduced as compared to LOCBH lasers that we have fabricated. Figure 3 shows lateral far-field patterns for ISBH lasers with active widths of 2.5 and $4 \mu$. Although the LOC waveguide structure can support two modes in the direction perpendicular to the junction, ISBH lasers typically operated in the fundamental mode in the direction perpendicular to the junction. The full width at halfmaximum of the far field in this direction was typically 30 $35^{\circ}$.

We have also investigated the effect of varying the aluminum content of the $\mathrm{Al}_{y} \mathrm{Ga}_{1-y}$ As burying layer. The modal characteristics were found to be much less sensitive to the
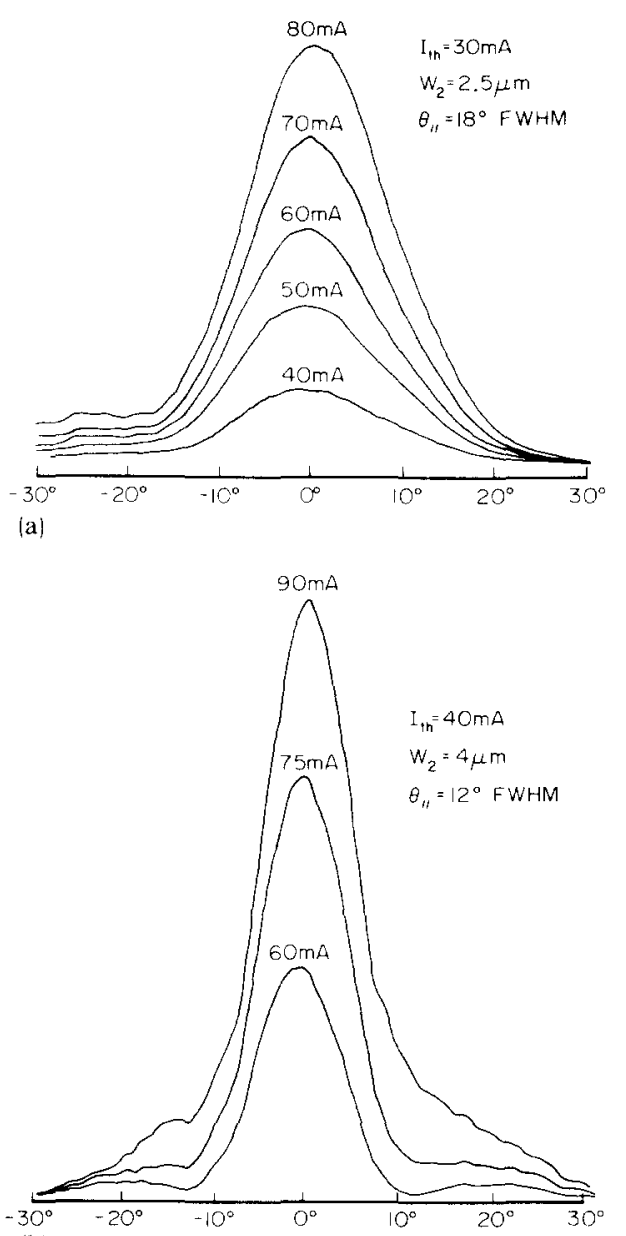

(b)

FIG. 3. Lateral far-field patterns of ISBH lasers.

aluminum content of the burying layers than were conventional LOCBH lasers, although increasing the aluminum content $y$ of the burying layer from 0.3 to 0.6 decreased the maximum stripe width for which fundamental mode operation was obtained from 4 to $3 \mu \mathrm{m}$. However, the lasers with $y=0.6$ had significantly improved thermal characteristics. Similar results have recently been reported for conventional BH lasers with high aluminum content burying layers. ${ }^{4} \mathrm{La}$ sers with $y=0.6$ operated $\mathrm{cw}$ at output powers up to the catastrophic optical damage limit even when mounted junction up. The temperature dependence of the pulsed threshold current for ISBH lasers is shown in Fig. 4.

Recently, we reported the fabrication of large optical cavity buried heterostructure window lasers in which the active layers did not extend to the mirrors. ${ }^{5}$ These lasers were operated at powers up to $130-\mathrm{mW} / \mu \mathrm{m}$ stripe width per facet. This was three times the power density at which otherwise identical lasers in which the active layer extended to the mirrors failed by catastrophic mirror damage. Since the output power was limited by heating, even larger power densities should be possible from this structure by improving the thermal characteristics of these lasers. In the LOCBH window lasers, fundamental mode operation was achieved only for stripe widths up to $2 \mu \mathrm{m}$ and the far fields were frequently 


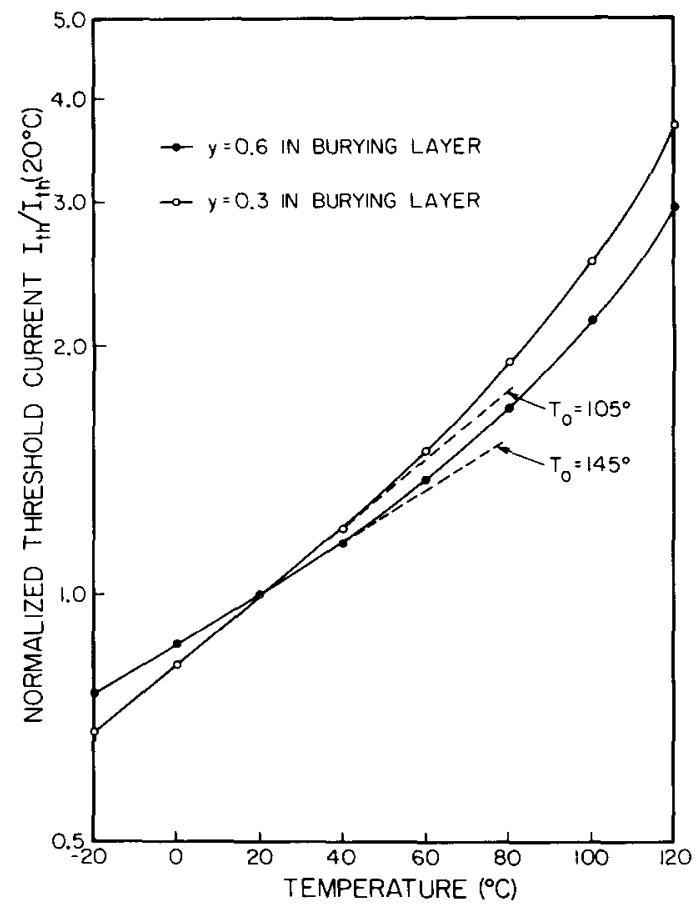

FIG. 4. Temperature dependence of the pulsed threshold current of ISBH lasers.

highly irregular due to scattered light. However, the window laser technology is completely compatible with the ISBH laser structure and we believe the application of the window technology to the ISBH structure will result in significant improvements in output power and beam quality as compared to the LOCBH window lasers.

In conclusion, we have fabricated inverted strip buried heterostructure lasers which have threshold currents and quantum efficiencies that are comparable to those of conventional LOCBH lasers. However, predominantly fundamental transverse mode operation of ISBH lasers was obtained for stripe widths up to $4 \mu \mathrm{m}$ which was twice as wide as we were able to obtain fundamental mode operation in LOCBH lasers. We also found the beam quality of ISBH lasers to be significantly improved as compared to LOCBH lasers which we have fabricated. Finally, the ISBH laser structure is compatible with a recently developed window laser technology. Fabrication of ISBH window lasers may result in significant improvements in the output power and beam quality over that obtained with LOCBH window lasers.

This work is supported by the Office of Naval Research and the National Science Foundation.

'N. Chinone, K. Saito, R. Ito, K. Aiki, and N. Shige, Appl. Phys. Lett. 35, $513(1979)$.

${ }^{2}$ H. Nakashima and K. Aiki, Jpn. J. Appl. Phys. 19, 591 (1980).

${ }^{3}$ W. T. Tsang and R. Logan, IEEE J. Quantum Electron. QE-15, 451 (1979).

${ }^{4} \mathrm{C}$. Henry, R. Logan, and F. Merritt, IEEE J. Quantum Electron. QE-17, 2196 (1981).

${ }^{5}$ H. Blauvelt, S. Margalit, and A. Yariv, Appl. Phys. Lett. 40, 1029 (1982). 